\title{
GÉNERO Y FORMACIÓN DE CAPITAL HUMANO: INICIATIVAS PÚBLICAS Y PRIVADAS en Málaga durante la crisis de la restauración
}

Concepción Campos Luque

Universidad de Málaga

El presente artículo tiene como objetivo fundamental poner de manifiesto las actuaciones llevadas a cabo por el Estado, las instituciones públicas o privadas y las sociedades obreras, para conseguir lo que hoy llamamos formación de capital humano, durante primer tercio del siglo XX en Málaga. La época histórica es especialmente interesante en este campo. Se caracteriza por la puesta en marcha de una política intervencionista que trata, sobre el papel, de regular las relaciones laborales y de establecer una normativa que facilite el acceso de la población a la enseñanza y a la formación profesional. Pero en la realidad las actuaciones son mínimas. Al analizar el fenómeno hacemos especial hincapié en la situación de la población femenina y su relación con la masculina.

The main goal of this paper is to study the human capital investment policies conducted by the Government, public and private institutions as well as unions during the first third of 20th century in Malaga. This historical period is especially relevant for this issue, as it is characterised by an increasing labour market intervention. The main objectives were the regulation of the labour relationships and the promotion of secondary and technical education. But in practice, the impact was negligible. In analysing this phenomenon, we focus on female population in comparison to the male population.

Palabras clave: Capital bumano, género, siglo XX. Málaga.

Key words: Human capital, gender, 20th. century, Málaga.

...¿En qué emplea el obrero las boras de descanso? ¿Adónde va?... Va el obrero al café más de lo que iba. Juega más de lo que jugaba.. jy adónde iré...? Si en vez de ir al café quiero ir a una escuela primaria o una escuela técnica, ¿encuentro esta escuela? En todos los países existen la Escuela profesional y la Universidad, abiertas al proletariado... ¿Dónde 
bay en España testimonios de este interés del Estado por la educación?...¿Qué posibilidades de instruirse, de formarse le dio y le da el Estado espaniol al obrero?..., (Marcelino Domingo, "Las ocho horas en España. Las razones del patrono y las razones del obrero", El Socialista, 9 de agosto de 1921).

Aunque en las teorias de Adam Smith ya estaba presente la idea de la importancia de la educación y formación de la mano de obra para el desarrollo económico, y la necesidad consecuente de un gasto público o privado en educación, no ha sido hasta muy recientemente que se ha formulado el concepto de capital humano por Shultz ${ }^{1}$. La inversión en capital humano es la base para el desarrollo de las economías capitalistas. Sin embargo, en las primeras etapas de la industrialización, caracterizadas por un bajo nivel tecnológico, la necesidad o conveniencia de gastar tiempo y dinero en la formación de la fuerza de trabajo no era tan evidente. Los bajos niveles de cualificación requeridos por el sistema productivo y el férreo liberalismo económico, desanimaban el desarrollo de una política educativa que alcanzara más allá de las clases medias. Y en el caso de las mujeres ni siquiera eso.

A lo largo del primer tercio del siglo XX tiene lugar en España un interés cada vez mayor por mitigar el analfabetismo que todavía afectaba a la mayor parte de la población. El incipiente desarrollo del intervencionismo estatal y su plasmación en la regulación de las relaciones laborales llegará también a este campo, imponiéndose normas que obliguen a la clase patronal a hacer posible la formación de la clase obrera, sobre todo debido a que aún se emplea masivamente mano de obra infantil. El proceso es lento, por lo que durante largo tiempo el retraso de España se agudiza, situándose en la línea de los países más deprimidos. Y aunque la formación de la mano de obra incide en el desarrollo económico, a su vez esa tarea formativa es imposible sin un cierto nivel de desarrollo, pues lo que anima a la cualificación profesional es la demanda del mercado.

Fue el Estado el que llevó a cabo la política de formación, aunque de manera poco organizada y contando con unos niveles de inversión mínimos. En 1920, los gastos del Estado en educación por habitante son: 3,37 pesetas en Madrid, 2,70 en Sevilla y 2,87 en Málaga ${ }^{2}$.

Sabemos que la tasa de alfabetización es un indicador de la formación de capital humano en las primeras etapas de desarrollo. En 1900, aún era analfabeta más del cincuenta por ciento de la población española. Pero en el análisis de la tasa de alfabetización es importante introducir la perspectiva del género. Como ha destacado Tortella, haciendo una síntesis de trabajos anteriores, en Economía se ha llega-

1. T. W. SHULTZ (ed.), Investment in Human Capital, Nueva York, Free Press, 1971.

2. Anuario Estadístico, 1920, p. 361. 
do a la conclusión de que la tasa de alfabetización determina los niveles de renta; a mayor alfabetización más renta. Pero alcanzado cierto nivel, cuanto menor sea la alfabetización femenina, más se reducirá la renta. Este mecanismo, dada la menor participación de las mujeres en el sector productivo, sólo se puede explicar por su influencia como transmisoras de conocimientos en la esfera familiar. El fenómeno tiene tal importancia que se refleja en el nivel de la renta de un país.

De esta manera podríamos decir que la alfabetización femenina actúa como un agente transmisor en la formación de la población de un país, al margen de las estructuras educativas. Este fenómeno -ampliamente conocido y debatido desde el punto de vista de la ideología, la socialización ${ }^{4}$, o la mentalidad, y que tradicionalmente ha determinado unas posturas ideológicas de restricción de la educación femenina por el temor a los cambios que se pudieran introducir en la familia y en la sociedad-, incorpora una vertiente económica no considerada, hasta muy recientemente, en toda su magnitud.

En España hay un 71,4\% de mujeres analfabetas en 1900, y algo más de la mitad de los hombres, mientras la media nacional se sitúa en torno al $60,9 \%$. Los avances durante la década de los años veinte son importantes, sobre todo para las mujeres, cuya tasa de analfabetismo desciende hasta llegar a ser en 1930 un $50 \% \%^{5}$.

En Málaga, el siglo se inicia con un más que dudoso honor. En 1910 la provincia arroja la tasa de analfabetismo más alta del país. Quedaba así claro, para vergüenza de las autoridades malagueñas, que un $75,83 \%$ de los hombres y un $82,99 \%$ de las mujeres eran analfabetas, lo que daba una tasa media de $79,46 \%$.

En el contexto andaluz Málaga venía presentando desde 1860 tasas globales de alfabetización inferiores; en general, ocupaba el quinto o el sexto lugar entre las provincias andaluzas. Pero a partir de 1910 se produce un empuje definitivo creciendo la tasa de alfabetización, hasta 1930, en 24,35 puntos?.

3. G. TORTELLA, El desarrollo de la España contemporánea. Historia económica de los siglos XIX $y^{\prime}$ XX, Madrid, Alianza, 1994, p. 41; Véase C. E. NUÑEZ ROMERO, La fuente de la riqueza. Educaciōn y desarrollo en la España contemporänea, Madrid, Alianza, 1992.

4. Un estudio del trabajo de socialización realizado por las mujeres y el diferente uso del tiempo entre mujeres y hombres en M. HAICAULT, "Socialisation domestique et reproduction sociale", en M. D. RAMOS y M. T. VERA (eds.), El trabajo de las mujeres. Pasado y presente, vol. 1, Actas del Congreso Internacional del Seminario de Estudios Interdisciplinares de la Mujer, Málaga, Diputación, 1996. pp. 53-63.

5. Ver R. M. CAPEL, "La educación de la mujer en el mundo contemporáneo", en Idem, El trabajo y la educación de la mujer en España (1900-1930). Madrid, Ministerio de Cultura (Instituto de la Mujer), 1986, pp. 302-503.

6. Le seguian Almeria, Jaén, Albacete y Murcia. Anuario Estadístico, Año 1917; ver F. MARTIN ZUÑGA, Origen, desarrollo y consecuencias del analfabetismo en el primer tercio del siglo XX. Análisis comparativo entre Málaga, Andalucia y España, Málaga, Universidad, 1992.

7. C. PELLEJERO, La filoxera en Málaga. Una crisis del capitalismo agrario andaluz, Málaga, Arguval, 1990, p. 152. 
Según los datos que recogemos en el cuadro 1, en 1910, la tasa de analfabetismo masculino en la capital es de 60,92\% y la femenina el $71,25 \%$. En 1920 , en la provincia todavía hay una tasa global de $73,04 \%$ de analfabetos, aunque la tasa femenina ha descendido a un $77,63 \%$ y la masculina a un $68,34 \%$. En 1920 , la tasa femenina en la capital es el $65,46 \%$ y la masculina el $53,09 \%$. La recuperación, aunque lenta, es clara; y los niveles de las tasas en la capital siempre más bajos que en el conjunto de la provincia ${ }^{8}$. Aunque la tasa femenina cae, la distancia con respecto a la masculina se acentúa. Este mismo fenómeno se produce en el conjunto del Estado.

Las reacciones y denuncias en la prensa, ante esta situación, se suceden, culpando a las autoridades municipales y al Estado del abandono en que se encuentra la población:

\begin{abstract}
"Según datos tomados de las estadísticas de las provincias de Andalucía, resulta que la de Málaga es la que mayor número de analfabetos tiene: un setenta y nueve por ciento. (...) ¿Qué males proceden del analfabetismo? ¡Infinidad de ellos! La incultura, la esclavitud, la adulación, la hipocresía, la vagancia, el lacayismo, el crimen, el robo, la explotación y el abuso del fuerte contra el débil. Toda tiranía se abre paso con la ignorancia. ¿Quién paga sus consecuencias? ¡La sociedad que trabaja y sufre! ¿Y quiénes son los que recogen el fruto de tanta ignorancia? ¿Los caciques y sus secuaces... Málaga ofrece un aspecto vergonzoso: los niños se ven a cualquier hora y por todas partes atropellando todo (...) La falta de escuelas y educación ha hecho y hará que en Málaga retroceda el progreso..."?.
\end{abstract}

Las soluciones al problema del analfabetismo proceden, como ya apuntábamos, de la puesta en marcha, por parte del Estado, de una política educativa que se remonta en sus líneas básicas al siglo XIX y la Ley Moyano, a partir de la cual se establece la enseñanza obligatoria hasta los doce años. Pero las dificultades económicas, así como la necesidad de emplear a menores, contribuyeron al incumplimiento sistemático de la legislación educativa que, al igual que en otro orden de cosas, es una característica de la época.

La situación de las escuelas era lamentable ${ }^{10}$. En 1915 la prensa denuncia que cada vez hay menos escuelas y menos maestros y que el presupuesto destinado a

8. La tasa global todavia era excesivamente alta en 1920. Málaga se considera la capital con mayor tasa de analfabetos del Estado. Según la Asociación de Magisterio Público de la capital, esto se debía a un error, por lo que envió un oficio al Ayuntamiento, en noviembre de 1922, "dando las gracias por la defensa que el Concejo ha hecho, con motivo de haberse considerado con manifiesto error a esta capital como la primera por el número de su analfabetismo". Boletin Oficial de la Provincia de Mälaga, 25 de diciembre de 1922.

9. R. MARIN TORNERO, "Las víctimas de la incultura", El Popular, 7 de marzo de 1919.

10. Sobre las condiciones de las escuelas E. BLANCO ALCANTARA, Higiene de las escuelas püblicas de Málaga. Conferencia leída en la Sociedad Española de Higiene, Málaga, La Española, 1904. Para la 
la enseñanza ha descendido ${ }^{11}$. Sin embargo, aunque el periodo histórico no destaca como una época de expansión de los centros de enseñanza, algunos años antes, concretamente desde 1909, había tenido lugar una cierta mejora de la fundación de colegios públicos, hecho que está íntimamente relacionado con las gestiones realizadas desde el gobierno municipal y por el delegado regio Narciso Díaz de Escobar, a lo que además contribuyó la circunstancia de que la cartera del Ministerio de Instrucción pública estuviera ocupada por Francisco Bergamín, así como por la capacidad gestora de Pedro Gómez Chaix ${ }^{12}$. Pero a partir de los años veinte se entra en una nueva dinámica que va a afectar muy directamente a las escuelas públicas.

Por su parte, la Iglesia en el marco histórico de la Restauración trata cle desarrollar todos los medios posibles para afianzar su control ideológico de la población a través de la enseñanza, en unos años que se caracterizan por una escalada del anticlericalismo ${ }^{13}$. De hecho la Iglesia en esta coyuntura recupera poder frente a los que defienden la escuela laica. Se ha dicho que el pacto entre la Restauración y la Iglesia, sellado por medio del monopolio de la educación, significa el fracaso del liberalismo en su afán de hacer frente a los problemas económicos que lastran su implantación de un modelo educativo, frente a la amplitud de medios de que dispone la Iglesia.

La institución eclesiástica fomenta la creación de escuelas dentro de dos ámbitos. Por un lado, la enseñanza de obreros/as y niños/as pobres, lo que entra en el campo de las actividades benéficas, no asumidas aún por el Estado. Y el de los colegios para la enseñanza y formación católica de los hijos/as de la clase media.

situación de las escuelas y la enseñanza ver: E. ORTEGA BERENGUER, La enseñanza en Málaga (18331933), Málaga, Universidad, 1985; un estudio detallado de la evolución de las escuelas en Málaga en F. MARTIN ZUÑIGA, La enseñanza primaria en Málaga durante el reinado de Alfonso XIII (1902-1931), Málaga, Diputación, 1993; Idem, "El desarrollo de la Instrucción pública en la Málaga de comienzos de siglo: Contribución de Narciso Díaz de Escobar desde la Delegación Regia de Primera Enseñanza (19091923)". Jábega, 68, 1990, pp. 49-58.

11. El Popular, 10 de mayo de 1915.

12. El interés y la vinculación de Gómez Chaix con el mundo de la enseñanza, del cual formó parte, entre sus otras muchas actividades, como profesor y secretario de la Escuela de Comercio, culmina con la creación el 21 de enero de 1923 de la Fundación Gómez Chaix, cuyo objetivo era crear un premio destinado a promover los estudios de Magisterio. Sobre la personalidad de Gómez Chaix véase: A. CABALLERO CORTES, Pedro Gómez Chaix: Director de la Económica malagueña (1906-1926). Mâlaga, Diputación, 1990. Sobre su labor política F. ARCAS CUBERO, El republicanismo malagueño en la Restauración (1875-1923), Córdoba, Ayuntamiento, 1985. Sobre su actividad como profesor de la Escuela de Comercio C. CAMPOS y A. MONTIEL, Los estudios mercantiles en Málaga. Proyectos y realidades, Málaga, Universidad, 1990.

13. E. de MATEO AVILES, Anticlericalismo en Málaga, 1874-1923, Málaga, Arguval, 1990; F. MARTIN ZUÑIGA, "La lucha contra la secularización de la enseñanza en las primeras décadas del siglo XX (1900-1930) vista desde el Boletín Oficial del Obispado de Málaga", Puerta Nueva, 17, 1993, pp. 61-70; A. MAYORDOMO PEREZ, Iglesia, Estado y Educación. El debate sobre la secularización de la enseñanza en España 1900-1913, Valencia, Rubio Esteban, 1982. 
En el conjunto de la enseñanza religiosa las escuelas parroquiales jugaron un papel fundamental. En 1917 el Obispo de Málaga ofrece una subvención para la creación de estas escuelas que se ubican preferentemente en los barrios obreros, como es el caso de Huelin donde se funda una escuela de niños y otra de niñas ${ }^{14}$. A pesar de la ayuda económica de la Iglesia las condiciones de estas escuelas no eran mucho mejores que las que solían ser frecuentes en las laicas:

“...el pavimento del patio y de la clase de niñas lo mismo puede servir para herir las rodillas de un cristiano que para pertrechos guerreros en las pedreas famosas de nuestros chaveas... y de cristales están allí tan escasos como abundantes de polvo, humo de fábricas y viento... aunque también es verdad que no son mejor acondicionadas las casa de estos pobres vecinos... lestas escuelasl albergan a más de un centenar de chiquillos y chiquillas peleando a brazo partido contra la roña presente y futura del cuerpo y del alma"15.

Por otro lado, Iglesia y Gobierno colaboran en la organización de enseñanzas nocturnas para niños y niñas de las clases populares, con un marcado carácter paternalista, a cargo de los Patronatos católicos.

El Regional, con un estilo fiel a su espíritu conservador, informa de la apertura de las clases organizadas por el Patronato de la Victoria, recomendando a los obreros que se apresuren a enviar a sus hijos e hijas:

“...para que se instruyan y eduquen. No os preocupéis del traje ni del calzado; al niño obrero le basta con ir limpio; muchos maestros, caritativamente, admiten a los niños aunque lleven roto el traje y descalzos".

Como premio a la asistencia el Patronato de la Victoria entrega:

“...centenares de alpargatas a todos los hijos de obreros de los barrios de Capuchinos, Malagueta y Victoria que en un mes no hayan faltado a clase. A finales de 1919 se repartieron cinco lotes de a 100 pesetas al niño o niña que se distinga por: Aplicación, Puntualidad, Virtud, Amor a los Maestros, Amor a los padres. Firmado: Un amigo de los niños obreros"16.

Cualquier comentario sería gratuito.

14. Estas escuelas serán católicas, eucaristicas, parroquiales, patrióticas y accesibles a los pobres "Condiciones que se comprometen a cumplir los Párrocos y Maestros que reciban subvención para escuelas parroquiales del Ilmo. Sr. Obispo Administrador Apostólico de Málaga", Boletín Oficial Eclesiástico de Málaga (BOEM), 15 de noviembre de 1917.

15. "Acción catequística. Hablemos de escuelas", BOEM, 15 de octubre de 1917.

16. El Regional, 1 de enero, 1919. 
Entre las actuaciones de la Iglesia encaminadas a dar formación profesional a las mujeres tenemos que destacar las actividades de El Instituto de la Inmaculada para el Servicio doméstico, fundado en Málaga en 1903 con dos fines fundamentales: proteger a las chicas que venían de los pueblos en busca de una ocupación como sirvientes, especialmente controlando su moral; y organizar escuelas nocturnas gratuitas para obreras en las que se les enseñará sus deberes de cristianas, además de labores, caligrafía, aritmética, y música. Sin duda, entre las trabajadoras del servicio doméstico se encuentran los mayores niveles de analfabetismo, por lo que la labor de la Iglesia en este caso era significativa. Aunque por los datos que hemos podido consultar, el número de asiladas en el Instituto no era muy elevado, así como tampoco lo debió ser el de asistentes a las escuela nocturna ${ }^{17}$.

La enseñanza primaria de las mujeres y niñas pobres queda en manos de las comunidades religiosas femeninas a falta de instituciones del Estado. Entre las más significativas se encuentran las monjas del Asilo de Jesús María y José de las Hermanas de la Caridad quienes, ayudadas por las 'damas del patronato', se ocupan de "...reformar a esos corazoncitos que... no han tenido más cuna ni más escuela, ni más educación que el arroyo". Estas monjas se encargan de las niñas llamadas 'colilleras' porque se dedican a recoger en la calle los desperdicios de los fumadores ${ }^{18}$. Las Adoratrices dan asilo a las prostitutas. En 1916 tienen aproximadamente cincuenta educandas. Estas mujeres además de ser adoctrinadas en la religión debían realizar "el trabajo de labores femeninas y de instrucción literaria". El Asilo de San Manuel se fundo para "la instrucción y ensenanza de pobrecitas huérfanas". En 1916 se educaban mil niñas y niños. En el Asilo de San Juan de Dios se alimenta y enseña a los niños pobres de la zona del Molinillo, en 1916 acogía a 1180 niños y niñas $^{19}$. En la Casa de la Misericordia se formaban profesionalmente a los niños y

17. J. GARCLA PEREZ, "Analfabetismo y servicio doméstico. Un intento de aproximación a la realidad cultural y actividad socioprofesional de la mujer extremeña en el primer tercio del siglo XX", Norba. Revista de Historia, 8-9, Cáceres 1987-88, pp. 215-241. Sobre el Instituto de la Inmaculada en Málaga AIMI, Fundaciōn de la Casa en Málaga, Documento mecanografiado, s/a; Idem, Reglamento de los asilos para sirvientas de la Congregación de Hermanas del senticio doméstico de 1911. Taller de Investigación JUM, Año del centenario 1900. Mecanografiado; Libros de Diarios manuscritos 1911 a 1919.

18. "Acción social de prensa y caridad: Las colilleras. Asilo de Jesús, José y María", BOEM, 1916. La falta de escolarización y la pobreza ocasionaba que muchos niños y niñas vivieran casi abandonados en la calle. Es frecuente encontrar referencias a este problema en la prensa de la época. El Inspector de sanidad J. Rosado explica el problema de las niñas colilleras con estas palabras:"...a todas horas del día pueden verse en las calles..., niñas pequeñas, cuya edad varia entre los siete y doce años, desde el atardecer hasta las veinticuatro es cuando (...) dan la jornada recia, acompañadas por la madre, la abuela, la tía o la 'industrial' que las contrata (...) ". J. ROSADO, Higiene social: Mendicidad, vagancia y otras enfermedades sociales, Málaga-Córdoba, Alcalá, 1924, pp. 66-67.

19. E. de MATEO AVILES, Paternalismo burgués y beneficencia religiosa en la Málaga de la segunda mitad del siglo XIX, Málaga, Diputación, 1985. 
niñas pobres para que trabajen y aprendan oficios. En el origen de las Casas de la Misericordia se encuentran las Casas de Socorro que se crearon con destino a los huérfanos desamparados y en ellas:

“...además de la primera enseñanza se proporcionará a los niños y niñas la de un arte, profesión u oficio, con arreglo a las aptitudes..., estableciendo o utilizando las fábricas o talleres posibles" ${ }^{20}$.

Dada la precariedad de medios, al margen de las actuaciones del Estado y de la Iglesia ${ }^{21}$, surgen otras iniciativas por parte de algunas empresas que costean colegio para los obreros, de las sociedades obreras y de otras instituciones que tradicionalmente habían asumido el coste de colegios de primera enseñanza, como la Sociedad Económica de Amigos del País. La creación de escuelas anejas a empresas no es común en Málaga. A pesar de que los patronos están obligados a permitir que los obreros menores de 14 años tengan tiempo de ir a la escuela, aún dentro del horario de trabajo ${ }^{22}$, esto normalmente no se cumple e incluso se trata de engañar en este sentido a los Inspectores de Trabajo ${ }^{23}$. En el cuadro 2 hemos recogido el número de infracciones por este concepto en Andalucía y España.

Con el objetivo de facilitar el acceso de los obreros a la enseñanza y suplir en parte el abandono en materia de enseñanza del Estado y el Ayuntamiento, se organizaron clases nocturnas a las cuales podían asistir los trabajadores una vez concluida su jornada laboral ${ }^{24}$.

20. J. ROSADO, op. cit., pp. 222-224.

21. M. VICO MONTEOLIVA, "Una Institución regeneracionista: la Academia de Santa Teresa de Málaga. Primera etapa (1914-1924)", en La educación en la España contemporánea. Cuestiones históricas, Madrid, Sociedad Española de Pedagogía, 1985, pp. 175-184. De la misma autora "La mujer en el Instituto Escuela de Málaga", en VV. AA., Mujer y educación en España, 1886-1975, Actas del VI Coloquio de Historia de la Educación, Santiago, Universidad, 1990. pp. 555-566.

22. "Establece la Ley que el patrono está obligado a facilitar instrucción primaria y religiosa a los menores de catorce años, dedicando a ello una hora por la mañana y otra por la tarde. Este precepto legal es letra muerta en la práctica, y muy difícil de comprobar por los inspectores"., J. GONZALEZ de CASTRO, "El trabajo de la mujer en la industria", BIRS, tomo XI, sept. 1914, pp.318.

23. A pesar de las dificultades los Inspectores de Trabajo solían reseñar si los trabajadores sabian o no leer y escribir y si se les permitía asistir a la escuela. Podemos citar a modo de ejemplo el resultado de la Inspección de la empresa de Cajas y Estuches San Andrés de Málaga. El inspector, que tampoco debió asistir mucho a la escuela, señala que los menores (todas mujeres o niñas) "no saben leer ni escrivir (sic) no se les hesije (sic) la asistencia al colegio de instrucion (sic)...", Relación de las Inspecciones realizadas por la Comisión de la Junta Local de Reformas Sociales de Málaga, 29 de abril de 1909, AHMM, Secc. Reformas Sociales.

24. Sobre las escuelas de adultos en Mălaga: M. MORALES MUÑOZ, "Instrucción y cultura obrera en Málaga (1868-1873)", Jábega, 54, 1986, pp. 59-63; M. V. MARTIN RUIZ, "Las escuelas de adultos en Málaga:(1876-1902)", Jábega, 42, 1983; F. MARTIN ZUÑIGA, La enseñanza de adultos desde, 1900 a 1931: un caso de política educativa sexista, Málaga, Edinford, 1993. 
Algunas de estas iniciativas surgieron en el seno del republicanismo y se referían especialmente a la enseñanza de mujeres y niñas. En Málaga, en el año 1914 el Centro Republicano Federal costea un Colegio en calle Biedmas $n^{\circ} 4$, que organiza clases nocturnas para hijas de los trabajadores de ocho a diez de la noche:

“...a fin de que los padres obreros puedan contar con un centro donde a más de las faenas domésticas, puedan sus hijas adquirir el más indispensable grado de instrucción de que tan necesitada se encuentra la mujer proletaria" ${ }^{25}$.

Las sociedades obreras, conscientes de la necesidad de contribuir a la formación de la clase trabajadora, también van a organizar enseñanzas a las que puedan asistir las personas adultas. Socialistas y anarquistas tendrán entre sus objetivos la transmisión de conocimientos como una clave para la concienciación y emancipación de los trabajadores. Las sociedades anarquistas abogaban por las escuelas racionalistas como un arma para sustraer a la clase obrera de la influencia de la enseñanza confesional ${ }^{26}$. En los Reglamentos de las sociedades obreras creadas durante estos años, sobre todo en las anarquistas, se puede leer como objetivo: "...la creación de escuelas racionalistas para dar una educación sana y racional a los hijos de los trabajadores"27.

Otras iniciativas responden a la necesidad de facilitar el acceso a la educación de las mujeres al margen de la enseñanza pública. Una de ellas es la adoptada por el Ateneo Popular para la educación de señoritas "El Progreso". En una carta dirigida al Director de El Popular se expone la necesidad de la educación femenina, para la que se ofrecen facilidades económicas:

"En este orden ocupa sin lugar atención (sic), la educación e instrucción de la mujer... se hará una rebaja de la cuota añadiendo los beneficios siguientes. Todo socio con cuota de una peseta al mes tiene derecho previo pago de otra peseta mensual a educar en el Colegio que este centro sostiene a una de sus hijas (o hijos menores de seis años). Para educar a dos hijas abonará además 1,50 mensual y por tres hijas tres pesetas".

25. El Popular, 29 de septiembre de 1914

26. J. L. GUEREÑA y A. TLANA FERRER, Clases populares, cultura y educación. Siglos $X I X$ y $X X$, Madrid, UNED, 1990; A. MAYORDOMO PEREZ, Educación y cuestión obrera en la España contemporánea, Valencia, Naus Llibres, 1981; M. SAMANIEGO BONEU, Los movimientos obreros, la educación popular, Madrid, Narcea, 1982; J. RODRIGUEZ GUERRA, "Concepto y naturaleza de la educación en el PSOE a principios de siglo", Historia de la Educación, 5, 1986, pp. 351-358; C. E. LIDA, "Educación anarquista en la España del siglo XX", Revista de Occidente, 97, 1971, pp. 33-37; R. M. MADRID CALZADA, "La educación de las clases populares sevillanas: 1900-1975", en C. ARENAS (coord.), Industria y clases trabajadoras en la Sevilla del siglo XX, Sevilla, Universidad, 1995, pp. 183-198.

27. AGCM, Reglamento del Sindicato del Ramo del Vestir, Doc. mecanografiado. 
Las materias impartidas en el Colegio del Ateneo Popular eran: Lectura, Caligrafía, Aritmética, Gramática Castellana, Historia Universal y de España, Geometría, Geografía General y Particular de España, Higiene, Urbanidad, Moral y Labores $^{28}$.

Evidentemente, a pesar de la organización de la enseñanza nocturna y de la obligatoriedad de que los patronos facilitaran la asistencia de las obreras a clase, en la realidad, y según los datos numéricos antes analizados, la repercusión de estas medidas sobre la educación de la clase obrera, y en particular de las mujeres fue muy escasa ${ }^{29}$. Todavía en 1924 , como puede verse en los porcentajes obtenidos en el cuadro 3, más del 65\% de las trabajadoras del comercio y la industria malagueña son analfabetas, mientras que los hombres representan un $33,49 \%$ de analfabetos en el comercio y un $52 \%$ en la industria.

En cuanto a la enseñanza práctica de un oficio, ésta aún se basa fundamentalmente en la transmisión de conocimientos dentro del lugar de trabajo, de maestras a oficialas y de éstas a las aprendizas, manteniéndose un sistema más acorde con una economía preindustrial; mientras que los conocimientos necesarios para realizar las tareas de los trabajos de la aguja, que caracterizan al sistema doméstico, se realizan básicamente en el ámbito familiar. El desarrollo de la industrialización pone definitivamente en crisis este sistema de iniciación a un oficio. Surge la necesidad de regular el contrato de aprendizaje, y se hace por la Ley de 17 de julio de 1911, referido a la industria, comercio y actividades agrícolas. El patrono se obliga en el contrato a enseñar prácticamente al aprendiz, mediando o no retribución, y por un tiempo determinado ${ }^{30}$. Parece que el carácter excesivamente restrictivo de estos contratos, así como la no existencia de normas que regularan la cuantía a percibir por los aprendices, tuvieron como consecuencia que el número de contratos de aprendizaje fuera muy exiguo.

A partir de 1914 la instrucción primaria se va complementando, en algunos casos, con una formación profesional específica. Pero hasta los años veinte no se generaliza el acceso a la instrucción básica -lo que se denominaba las cuatro reglaspara las mujeres de las áreas urbanas, hecho que contribuyó a que mejorara la calidad de la mano de obra femenina y a que se sentaran las bases para recibir una

28. El Popular, 16 de diciembre de 1915.

29. En 1918 la Comisión permanente de la Asociación Nacional de Magisterio protesta por la supresión de las clases nocturnas para adultos durante aquel curso. El Regional, 7 de noviembre de 1918; Ver: F. MARTIN ZUÑIGA, "Escolarización y trabajo de la mujer malagueña en el primer tercio del siglo XX", El Trabajo de las mujeres. Pasado y Presente, op. cit.; F. GOMEZ RODRIGUEZ, "La educación general y la formación para el trabajo como vías separadas en la educación tradicional", Revista Española de Pedagogia, 138, 1977, pp. 415-436.

30. Para las condiciones y normas establecidas en los contratos de aprendizaje ver A. SOTO GARMONA, El trabajo industrial en la España Contemporánea, Barcelona, Anthropos, 1989, pp. 222-225. 
formación profesional ${ }^{31}$. Aunque el inspector del trabajo González de Castro pensaba que las obreras no necesitan mucho aprendizaje pues "las facultades de asimilación que posee la obrera española son extraordinarias en cualquier oficio, necesitando muy pocas lecciones y escaso aprendizaje para obtener brillantes resultados"32.

Tenemos datos de la proporción de obreras inclustriales malagueñas en cada una de las tres categorías profesionales clásicas en 1924, referidos a la industria textil, alimentación, libro y vestido, cuadro 4. Como podemos ver ninguna mujer alcanza la categoría de maestra en ninguno de los sectores industriales considerados, al tiempo que más del $90 \%$ en todos ellos se encuentran clasificadas en la categoría de oficialas, y una proporción muy pequeña en aprendizas, destacando en esta última la de la industria del vestido con $8,87 \%$. Sin embargo, para alcanzar la categoría de oficiala no era necesario haber tenido ninguna instrucción profesional, como se desprende del cuadro 5 , en el que hemos obtenido los porcentajes de obreras $\backslash$ os industriales con instrucción profesional. Las cifras son contundentes, el $100 \%$ de las mujeres no han recibido instrucción y el $99,84 \%$ de los hombres tampoco. La situación aún es más drástica en el sector del comercio en el que el $100 \%$ no han recibido instrucción.

En 1921 se edita un reglamento del Ateneo comercial fundado como sección de la Sociedad Económica de Amigos del País -que tradicionalmente había desarrollado un gran interés por crear y sostener escuelas de primeras letras desde el siglo XVIII $^{33}$-, entre cuyas actividades se contemplaba establecer Escuelas de Aprendices. Sin embargo, la falta de documentación sobre estas enseñanzas nos hace pensar que la idea no fue más allá del papel ${ }^{34}$.

Por otro lado, la participación de las mujeres en Institutos de Enseñanza Media, Industrial, Artes y oficios o Escuela de Comercio todavía es muy escasa y relegada de determinadas enseñanzas. El cuadro 6 muestra la evolución de los porcentajes globales, respectivamente, de primera y segunda enseñanza, pudiéndose comprobar que las mujeres pasan de representar un $19,32 \%$ de la población escolar total en 1910 a un $39,55 \%$ en 1920.

31. A. SOTO CARMONA, El trabajo ..., op. cit., p. 217; J. GAliCla AYALA, Memoria del Instituto general y técnico de Málaga del curso académico de 1912 a 1913 por ..., Málaga, Imp. de La Española, 1913.

32. op. cit., p.322.

33. Sobre las enseñanzas creadas por la Sociedad Económica y su rivalidad con las sostenidas por el Consulado de Comercio de Málaga véase C. CAMPOS y A. M. MONTIEL, "En el centenario de la Cámara de Comercio, Industria y Navegación de Málaga", Jábega, 52, 1986, pp. 39-48.

34. Reglamento del Ateneo Comercial de la Sociedad Económica de Amigos del País, Málaga, 1921. Entre las funciones y objetivos del Ateneo Comercial estaban: 1) Sostener Cátedras, impartir conferencias y cursos; 2) Crear bolsas de trabajo y agencias u oficinas de colocación; 3) Constituir sociedades cooperativas para la construcción de casa baratas; 4) Fundar Cajas de ahorro populares de auxilio destinadas a retiros para obreros y de mutualidad materna; 5) Establecer una escuela de aprendices. 
En 1914 la Escuela de Artes y Oficios de Málaga cuenta con una sección específica de Enseñanza de la mujer, en la que se impartía dibujo lineal y adorno y dibujo artístico. No había ninguna mujer en modelado, vaciado, composición o pintu$\mathrm{ra}^{35}$. En el curso 1918-1919 hay 34 mujeres matriculadas en artes y oficios ${ }^{36}$.

En la Escuela de Comercio ${ }^{37}$ la matrícula de mujeres se va haciendo cada vez más normal, aunque todavía son muy pocas las que estudian la carrera en comparación con los hombres. En 1915 se somete a informe de la Comisión de Hacienda un oficio del director de la Escuela Superior de Comercio de Málaga para arrendar una casa de calle Beatas con destino a la Sección Elemental femenina ${ }^{38}$. Aunque durante el curso 1918-19 hay matriculados 294 hombres y sólo 14 mujeres que se incrementaron hasta 20 en el curso siguiente ${ }^{39}$.

Las mujeres de clase media acceden durante estos años a nuevas ocupaciones: empleadas, dependientas, mecanógrafas, telefonistas o empleadas en la Administración del Estado, que les abrió sus puertas en $1918^{40}$.

En el caso de las empleadas de oficina particulares o de la Administración, uno de los escollos principales era la falta de preparación:

"Lo importante es que sepan todas las mujeres... que es indispensable la elevación de su cultura para poder penetrar airosamente en los modernos campos de trabajo a que se la invita"41.

Surgen academias particulares de mecanografía para mujeres, como la establecida por la casa Underwood, sucursal de la Compañía mecanográfica de Barcelona ${ }^{42}$. También la asociación de dependientes de comercio en su propaganda para lograr la asociación de las dependientas se declaran:

35. El Popular, 25 de octubre de 1914; C. ALVAREZ DUMONT y P. QUINTERO ATAURI, Escuela de Artes e Industrias de Málaga. Memoria dirigida a cuantos se interesan por la instrucción del obrero, Málaga, 1903; C. ALVAREZ DUMONT, Memoria reglamentaria del curso de 1911 a 1912 de la Escuela de Artes y Oficios de Málaga, Mălaga, Imp. de Zambrana, 1913.

36. Anuario Estadístico, 1919, pp. 428-429.

37. Un estudio de la evolución de las enseñanzas mercantiles en Málaga y de la Escuela de Comercio hasta 1900, en C. CAMPOS y A. MONTIEL, Los estudios mercantiles en Málaga..., op. cit.

38. Boletín Municipal de Málaga, 15 de diciembre de 1915.

39. Anuario Estadístico, 1919, p. 420.

40. G. FRANCO RUBIO, La incorporación de la mujer a la Administración del Estado, Municipios y Diputaciones: 1918-1936, Madrid, D.G.J., 1981.

41. S. LUENGO, "Para las mujeres", La Uniōn Mercantil, 8 de abril de 1921.

42. La Unión Ilustrada, 30 de noviembre de 1921, publica fotos de las mecanógrafas del despacho y de la academia. 
“...entusiasmados con la conducta de sus compañeras...piden que empiecen a funcionar una academia especiàl en la que las señoritas afiliadas puedan adquirir cuántos conocimientos sean necesarios para poder desempeñar airosamente su cometido" ${ }^{43}$.

Sin embargo, en la documentación que hemos consultado sobre la cualificación profesional de empleadas de oficinas y mecanógrafas, referidos a 1923, se consigna que ninguna de ellas había recibido instrucción profesional ${ }^{44}$.

Las escuelas de magisterio significaban casi la única oportunidad en las que se contemplaba abiertamente la enseñanza profesional de las mujeres. Estos centros constituyeron, en muchos casos, la única institución que dinamizaba la vida cultural de la ciudad ${ }^{45}$. Pero los estudios de magisterio eran poco exigentes y el peso de la religión excesivo. Básicamente el examen de ingreso se reducía a una pregunta de doctrina cristiana, otra de aritmética y gramática, dictado, lectura y una labor de costura. Esta situación se mantuvo durante el primer tercio del siglo $\mathrm{XX}^{46}$.

Por último, el trabajo de comadrona, matrona o profesoras de partos, como se las conocía en el ámbito profesional, requiere cualificación profesional a medida que el acto de parir se va considerando un acto clínico. En Málaga no existía escuela de matronas durante estos años por lo que éstas tenían que desplazarse para realizar sus estudios a la Universidad de Granada. Para obtener el título de matrona había que cursar los dos años que, a tal efecto, se impartían en la Facultad de Medicina ${ }^{47}$.

43. El Popular, 24 de octubre de 1919.

44. Relación de fichas de individuos de profesiones liberales que prestan servicio en empresas particulares, AHPM, Dirección General de Estadistica. Sección Provincial de Málaga.

45. M. J. RIVERA SANCHEZ, Historia de las Escuelas Normales de Málaga. Unas instituciones para la formación de los Maestros y maestras, 1846-1939. tesis doctoral. Universidad de Málaga, 1991.

46. En 1894 se creó en Málaga el centro pedagógico gratuito, institución para la enseñanza de la mujer, a instancias de Ana María Solo de Zaldivar, directora de la Normal, con el objetivo fundamental de suplir las deficiencias de la Escuela. Ver C. SANCHIDRIAN BLANCO, "El centro pedagógico gratuito de Mâlaga: una Institución para mejorar la formación de las maestras a finales del siglo XIX", Historia de la Educación. Revista Interuniversitaria, 6, 1987, pp. 249-259: Idem. "La formación de las maestras de Málaga hace un siglo. Aproximación a la historia de una tradición", VV. AA., Mujer y educación en Espa$\tilde{n} a, 1886-1975$, Actas del VI Coloquio de Historia de la Educación, Santiago, Universidad, 1990; Politica educativa y enseñanza primaria en Málaga durante la Restauración (1874-1902), Málaga, Universidad, 1986; A. CABALLERO, "La presencia de la mujer en la enseñanza en Málaga en la primera mitad del siglo XX", en Mujer y educación, op. cit., pp. 381-387.

47. Es frecuente que las mujeres solicitaran ayudas al Ayuntamiento para costear la matrícula o el título en la enseñanza de magisterio y de matronas. Citamos una a modo de ejemplo: "la que suscribe, (...), natural de esta ciudad, de estado soltera... Expone: Que habiéndose examinado en Granada...y habiendo obtenido nota favorable, resulta que no dispone de recursos pecuniarios... para costearse el título... por ello suplica... se sirvan acordar costearle sólo y exclusivamente el título de profesora... Málaga, 7 de octubre de 1924", solicitud que el ayuntamiento acordó desestimar. AHMM, leg. 3654, Matronas. 
En conclusión, a pesar de que se están operando algunos cambios que tienden a mejorar la educación y formación de la clase obrera, es patente que aún no se considera como un asunto prioritario por parte del Estado. Aunque ya se había legislado desde mediados del XIX estableciendo la obligatoriedad de la enseñanza hasta los doce años, es evidente que la legislación no se cumple. En Málaga, como hemos puesto de manifiesto, se denuncia constantemente durante estos años el abandono en que se encuentran los niños y las niñas y la falta de escuelas. La escasa inversión del Estado hace recaer en los Ayuntamientos la organización de la enseñanza, aunque poco pueden hacer, dado el exiguo presupuesto, más que atender al mantenimiento de las escuelas existentes, que en la mayoría de los casos se encuentran en unas condiciones higiénico-sanitarias deplorables. Todo ello determina que surjan otras iniciativas entre los republicanos, sociedades obreras o empresas. Aunque en Málaga no son muchas las actividades que se pueden documentar a este respecto. Republicanos y anarquistas, según la fuentes, parece que fueron los más preocupados por facilitar el acceso de las mujeres obreras a la enseñanza. Los anarquistas, en tanto que partidarios de las escuelas racionalistas, fomentaron la coeducación de niños y niñas.

En conjunto, podemos concluir que se dieron escasas oportunidades para la formación de las mujeres trabajadoras, a excepción de algunas actuaciones esporádicas.

A pesar de todos los problemas, los años estudiados significaron una mejora si tenemos en cuenta los elevados niveles de analfabetismo que se mantenían en Málaga a principios de siglo. La tasa de analfabetismo femenino desciende pero continúa siendo más elevada que la masculina.

En cuanto a la formación profesional poco nuevo se puede añadir. El acceso de las mujeres a este tipo de enseñanza, escasamente implantada en general, es prácticamente inexistente. Las trabajadoras se encuentran en las escalas inferiores de la cualificación profesional. Ser aprendiza u oficiala, analfabeta y con escasa o nula cualificación constituye el perfil general de la obrera malagueña durante los años estudiados.

Por otra parte, se produce un incremento de alumnas en las enseñanzas medias y una tímida incorporación a otras enseñanzas como Comercio y Artes y Oficios, aunque en el último caso en asignaturas especialmente diseñadas para mujeres, existiendo una segregación clara en este sentido.

Para las trabajadoras la falta de educación y formación va a significar una forma más de desigualdad y discriminación dentro de la que caracteriza en la época a la clase obrera en su conjunto. Este es un factor más que incide en las condiciones de trabajo y de vida, cuya máxima repercusión consistirá en servir de argumento para justificar el menor salario de las mujeres.

El binomio ignorancia-probreza y la falta de oportunidades para superarlo, aunque forma parte consustancial de las condiciones de vida de la clase obrera, es más 
acentuado en las mujeres. La formación para el trabajo ha de encontrarse en el propio ámbito familiar o dentro de la empresas después de largos años de pasar por la categoria de aprendizaje escasa o nulamente retribuida. Permítasenos recordar un último dato: en 1924, el $65, \%$ de las mujeres que trabajan en el comercio y el $67,19 \%$ de la obreras industriales malagueñas son analfabetas.

Con este panorama de escasa inversión estatal e iniciativas particulares, poco o nada se podía transformar la cultura y formación profesional de la población en general, y menos la de las mujeres, factor por otro lado indispensable para el desarrollo económico del país. 
Cuadro 1.

Porcentajes de analfabetos/as. Málaga capital y provincia.

\begin{tabular}{l|c|c|c|c|c|c}
\hline & \multicolumn{3}{|c|}{ Capital } & \multicolumn{3}{c}{ Provincia } \\
\hline & 1900 & 1910 & 1920 & 1900 & 1910 & 1920 \\
Mujeres & 70,40 & 71,25 & 65,46 & 81,52 & 82,99 & 77,63 \\
Hombres & 70,75 & 60,92 & 53,09 & 74,49 & 75,83 & 68,34 \\
TOTAL & 65,85 & 66,34 & 59,62 & 78,06 & 79,46 & 73,04 \\
\hline
\end{tabular}

Elaboración propia. Fuente: Anuario Estadístico 1923-1924.

Cuadro 2.

Empresas que no facilitan instrucción primaria y religiosa

\begin{tabular}{l|r|r|r|r|r}
\hline \multicolumn{2}{c|}{ Andalucía } & \multicolumn{3}{c}{ España } \\
\hline Provincias & \multicolumn{1}{c|}{ I } & II & Regiones & I & II \\
\hline Granada & 762 & 301 & $1^{\mathrm{a}}$ & 239 & 2.563 \\
Almería & 40 & 16 & $2^{\mathrm{a}}$ & 709 & 2.526 \\
Cádiz & 20 & 20 & $3^{\mathrm{a}}$ & 425 & 1.568 \\
Córdoba & 679 & 105 & $4^{\mathrm{a}}$ & 544 & 1.627 \\
Huelva & 10 & 8 & $5^{\mathrm{a}}$ & 3.096 & 1.417 \\
Jaén & 16 & 5 & $6^{\mathrm{a}}$ & 258 & 2.043 \\
Málaga & 534 & 624 & $7^{\mathrm{a}}$ & 320 & 915 \\
Sevilla & 1.035 & 338 & $8^{\mathrm{a}}$ & 1.531 & 673 \\
& & & Baleares & & 262 \\
& & & Canarias & 79 & 220 \\
\hline Total & 3.096 & 1.417 & & 79.791 & 13.814 \\
\hline
\end{tabular}

Total general de infracciones: 201.219

I. Número de infracciones.

II. Número de empresas visitadas.

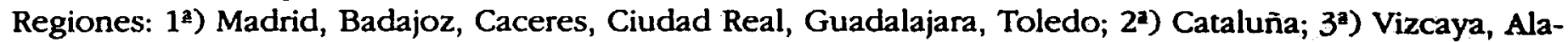
va, Guipuzcoa, Logroño, Santander; 4a) Oviedo, Coruña, León, Lugo, Orense, Pontevedra; 5ª) Andalucía; 6a) Valencia, Albacete, Alicante, Castellón, Cuenca, Murcia; 7a) Salamanca, Avila, Burgos, Valladolid, Zamora; 8a) Zaragoza, Huesca, Navàrra, Teruel.

Elaboración propia

Fuente: Memoria General de la Inspección de Trabajo 1912, IRS, Madrid, Minuesa, 1914, pp. 394-405 
Cuadro 3.

Instrucción elemental. Málaga capital 1924. Porcentajes.

\begin{tabular}{l|c|c|c|c}
\hline & \multicolumn{2}{|c|}{ Comercio } & \multicolumn{2}{c}{ Industria } \\
\hline & Hombres & Mujeres & Hombres & Mujeres \\
\hline Saben leer y escribir & 65,84 & 34.15 & 47.7 & 32,60 \\
Saben sólo leer & 0,67 & 0 & 0,3 & 0,21 \\
No saben leer ni escribir & 33,49 & 65,85 & 52 & 67,19 \\
TOTAL & 100 & 100 & 100 & 100 \\
\hline
\end{tabular}

Elaboración propia. Fuente: AHPM, leg. E-37.

\section{Cuadro 4.}

Porcentajes de mujeres por industrias y categorias profesionales. Málaga capital. 1924

\begin{tabular}{l|c|c|c|c}
\hline & Aprendizas & Oficialas & Maestras & Total \\
\hline Textiles & 6,40 & 93,60 & 0 & 100 \\
Alimentación & 1,56 & 98,44 & 0 & 100 \\
Libro & & 100 & & 100 \\
Vestido & 8,87 & 91,13 & 0 & 100 \\
\hline
\end{tabular}

Elaboración propia.

Fuente: AHPM, Leg. E-37.

Cuadro 5.

Intrucción profesional. Málaga capital, 1924. Porcentajes.

\begin{tabular}{l|c|c|c|c}
\hline & \multicolumn{2}{|c|}{ Comercio } & \multicolumn{2}{c}{ Industria } \\
\hline & Hombres & Mujeres & Hombres & Mujeres \\
\hline Lo han recibido & 0 & 0 & 0,16 & 0 \\
No lo han recibido & 100 & 100 & 99,84 & 100 \\
TOTAL & 100 & 100 & 100 & 100 \\
\hline
\end{tabular}

Elaboración propia. Fuente: AHPM, leg. E-37 
Cuadro 6.

Málaga capital. Población escolar. Porcentajes.

\begin{tabular}{c|c|c|c}
\hline & Mujeres & Hombres & Total \\
\hline 1910 & 19.32 & 80.67 & 100 \\
1920 & 39,55 & 60,45 & 100 \\
\hline
\end{tabular}

1910 Porcentajes sobre los datos totales que incluyen alumnos de primera enseñanza, segunda enseñanza, Facultades y carreras especiales.

1920 Porcentajes sobre los datos totales de alumnos de escuelas y colegios de primera enseñanza y estudiantes.

Elaboración propia. Fuente: Censos 1910 y 192 